\title{
Application of Maple on the Limit Problems
}

\author{
Chii-Huei Yu \\ Department of Information Technology, Nan Jeon University of Science and Technology, Taiwan
}

Copyright $\odot 2017$ by authors, all rights reserved. Authors agree that this article remains permanently open access under the terms of the Creative Commons Attribution License 4.0 International License

\begin{abstract}
This paper uses the mathematical software Maple to study four types of limit problems. The solutions of the four types of limits can be obtained mainly using complex function method. On the other hand, we propose some examples to demonstrate the calculations. The research method adopted in this study is to find solutions through manual calculations and verify the answers using Maple. This method can not only allow the discovery of calculation errors, but also help modify the original directions of thinking.
\end{abstract}

Keywords Limit Problems, Complex Function Method, Maple

\section{Introduction}

The computer algebra system (CAS) has been widely employed in mathematical and scientific studies. The rapid computations and the visually appealing graphical interface of the program render creative research possible. Maple possesses significance among mathematical calculation systems and can be considered a leading tool in the CAS field. The superiority of Maple lies in its simple instructions and ease of use, which enable beginners to learn the operating techniques in a short period. In addition, through the numerical and symbolic computations performed by Maple, the logic of thinking can be converted into a series of instructions. The computation results of Maple can be used to modify our previous thinking directions, thereby forming direct and constructive feedback that can aid in improving understanding of problems and cultivating research interests. The introduction of Maple can refer to [1-4].

In calculus and engineering mathematics, there are many ways to solve the limit problems, the discussion of limit problems can be referred to [5-7]. But for the more difficult limit problems, the use of calculus approach is not necessarily easy to get the answer. So this article uses the complex function method to solve the limit problems. The followings are the four types of limit problems discussed in this paper:

$$
\begin{aligned}
& \cos 5 \theta \cdot \sin (\operatorname{srcos}(\theta+\varphi)) \cdot \cosh (\operatorname{sr} \sin (\theta+\varphi)) \\
& +\sin 5 \theta \cdot \cos (s r \cos (\theta+\varphi)) \cdot \sinh (s r \sin (\theta+\varphi)) \\
& \lim _{r \rightarrow 0} \frac{-s r \cos (4 \theta-\varphi)+\frac{1}{6} s^{3} r^{3} \cos (2 \theta-3 \varphi)}{r^{5}}, \\
& \cos 5 \theta \cdot \cos (s r \cos (\theta+\varphi)) \cdot \sinh (s r \sin (\theta+\varphi)) \\
& -\sin 5 \theta \cdot \sin (s r \cos (\theta+\varphi)) \cdot \cosh (\operatorname{sr} \sin (\theta+\varphi)) \\
& \lim _{r \rightarrow 0} \frac{+s r \sin (4 \theta-\varphi)-\frac{1}{6} s^{3} r^{3} \sin (2 \theta-3 \varphi)}{r^{5}}, \\
& \cos 4 \theta \cdot \cos (s r \cos (\theta+\varphi)) \cdot \cosh (\operatorname{sr} \sin (\theta+\varphi)) \\
& -\sin 4 \theta \cdot \sin (s r \cos (\theta+\varphi)) \cdot \sinh (s r \sin (\theta+\varphi))] \\
& -1+\frac{1}{2} s^{2} r^{2} \cos (2 \theta-2 \varphi) \\
& \lim _{r \rightarrow 0} \frac{2}{r^{4}} \text {, } \\
& -\cos 4 \theta \cdot \sin (s r \cos (\theta+\varphi)) \cdot \sinh (\operatorname{sr} \sin (\theta+\varphi)) \\
& -\sin 4 \theta \cdot \cos (\operatorname{srcos}(\theta+\varphi)) \cdot \cosh (\operatorname{sr} \sin (\theta+\varphi)) \\
& \lim _{r \rightarrow 0} \frac{-\frac{1}{2} s^{2} r^{2} \sin (2 \theta-2 \varphi)}{r^{4}},
\end{aligned}
$$

where $r, s, \theta, \varphi$ are real numbers. Moreover, some examples are proposed, and we use Maple to calculate the approximation of these limits and their solutions to verify our answers.

\section{Materials and Methods}

First, some formulas used in this study are introduced below.

\subsection{Euler's Formula}

$e^{i \theta}=\cos \theta+i \sin \theta$, where $i=\sqrt{-1}$, and $\theta$ is a real number.

\subsection{De Moivre's Formula}

$$
(\cos \theta+i \sin \theta)^{p}=\cos p \theta+i \sin p \theta \text {, where } p \quad \text { is an }
$$


integer, and $\theta$ is a real number.

\subsection{Let $a, b$ Be Real Numbers, Then}

$$
\sin (a+i b)=\sin a \cosh b+i \cos a \sinh b,
$$

and

$$
\cos (a+i b)=\cos a \cosh b-i \sin a \sinh b .
$$

\subsection{If $z$ is a Complex Number, Then}

$$
\sin z=\sum_{k=1}^{\infty} \frac{(-1)^{k-1}}{(2 k-1) !} z^{2 k-1}=z-\frac{1}{3 !} z^{3}+\frac{1}{5 !} z^{5}-\cdots,
$$

and

$$
\cos z=\sum_{k=1}^{\infty} \frac{(-1)^{k-1}}{(2 k-2) !} z^{2 k-2}=1-\frac{1}{2 !} z^{2}+\frac{1}{4 !} z^{4}-\cdots
$$

To obtain the major results in this paper, the following lemma is needed.

Lemma Let $\lambda, z$ be complex numbers, then

$$
\lim _{z \rightarrow 0} \frac{\sin (\lambda z)-\lambda z+\frac{\lambda^{3}}{6} z^{3}}{z^{5}}=\frac{\lambda^{5}}{120},
$$

and

$$
\lim _{z \rightarrow 0} \frac{\cos (\lambda z)-1+\frac{\lambda^{2}}{2} z^{2}}{z^{4}}=\frac{\lambda^{4}}{24}
$$

$$
\begin{gathered}
\text { Proof } \lim _{z \rightarrow 0} \frac{\sin (\lambda z)-\lambda z+\frac{\lambda^{3}}{6} z^{3}}{z^{5}} \\
=\lim _{z \rightarrow 0} \frac{\left(\lambda z-\frac{\lambda^{3}}{3 !} z^{3}+\frac{\lambda^{5}}{5 !} z^{5}-\frac{\lambda^{7}}{7 !} z^{7}+\cdots\right)-\lambda z+\frac{\lambda^{3}}{6} z^{3}}{z^{5}} \\
=\lim _{z \rightarrow 0} \frac{\frac{\lambda^{5}}{120} z^{5}-\frac{\lambda^{7}}{5040} z^{7}+\cdots}{z^{5}}
\end{gathered}
$$

$$
=\lim _{z \rightarrow 0}\left(\frac{\lambda^{5}}{120}-\frac{\lambda^{7}}{5040} z^{2}+\cdots\right)
$$

$$
=\frac{\lambda^{5}}{120}
$$

On the other hand,

$$
\begin{gathered}
=\lim _{z \rightarrow 0} \frac{\cos (\lambda z)-1+\frac{\lambda^{2}}{2} z^{2}}{z^{4}} \\
=\lim _{z \rightarrow 0} \frac{\frac{\lambda^{4}}{24} z^{4}-\frac{\lambda^{5}}{120} z^{5}+\cdots}{z^{4}} \\
=\lim _{z \rightarrow 0}\left(\frac{\lambda^{4}}{24}-\frac{\lambda^{5}}{120} z+\cdots\right) \\
=\frac{\lambda^{4} \cdot}{24}
\end{gathered}
$$

In the following, we determine the limits of (1) and (2).

Theorem 1 Suppose that $r, s, \theta, \varphi$ are real numbers, then

$$
\begin{aligned}
& \cos 5 \theta \cdot \sin (\operatorname{srcos}(\theta+\varphi)) \cdot \cosh (\operatorname{sr} \sin (\theta+\varphi)) \\
& +\sin 5 \theta \cdot \cos (\operatorname{srcos}(\theta+\varphi)) \cdot \sinh (\operatorname{sr} \sin (\theta+\varphi))
\end{aligned}
$$$$
\lim _{r \rightarrow 0} \frac{-s r \cos (4 \theta-\varphi)+\frac{1}{6} s^{3} r^{3} \cos (2 \theta-3 \varphi)}{r^{5}}=\frac{s^{5} \cos 5 \varphi}{120}
$$

and

$$
\begin{aligned}
& \cos 5 \theta \cdot \cos (s r \cos (\theta+\varphi)) \cdot \sinh (s r \sin (\theta+\varphi)) \\
&-\sin 5 \theta \cdot \sin (s r \cos (\theta+\varphi)) \cdot \cosh (s r \sin (\theta+\varphi)) \\
& \lim _{r \rightarrow 0} \frac{+\operatorname{sr} \sin (4 \theta-\varphi)-\frac{1}{6} s^{3} r^{3} \sin (2 \theta-3 \varphi)}{r^{5}}=\frac{s^{5} \sin 5 \varphi}{120} .
\end{aligned}
$$

Proof In Eq. (9), let $z=r e^{i \theta}, \lambda=s e^{i \varphi}$, then by Euler's formula and De Moivre's formula, we have 


$$
\lim _{r \rightarrow 0} \frac{e^{-i 5 \theta} \sin \left(s r e^{i(\theta+\varphi)}\right)-s r e^{i(-4 \theta+\varphi)}+\frac{1}{6} s^{3} r^{3} e^{i(-2 \theta+3 \varphi)}}{r^{5}}=\frac{s^{5} e^{i 5 \varphi}}{120}
$$

Thus,

$$
\begin{aligned}
& (\cos 5 \theta-i \sin 5 \theta) \cdot \sin (s r \cos (\theta+\varphi)+i s r \sin (\theta+\varphi)) \\
-s r \cos (4 \theta-\varphi)+i s r \sin (4 \theta-\varphi) & =\frac{s^{5} \cos 5 \varphi+i s^{5} \sin 5 \varphi}{120} . \\
\lim _{r \rightarrow 0} \frac{+\frac{1}{6} s^{3} r^{3} \cos (2 \theta-3 \varphi)-i \frac{1}{6} s^{3} r^{3} \sin (2 \theta-3 \varphi)}{r^{5}} &
\end{aligned}
$$

Using Eq. (5) yields

$$
\begin{aligned}
& (\cos 5 \theta-i \sin 5 \theta)[\sin (s r \cos (\theta+\varphi)) \cdot \cosh (s r \sin (\theta+\varphi)) \\
& +i \cos (s r \cos (\theta+\varphi)) \cdot \sinh (s r \sin (\theta+\varphi))] \\
& -s r \cos (4 \theta-\varphi)+i s r \sin (4 \theta-\varphi) \\
\lim _{r \rightarrow 0} \frac{}{6} s^{3} r^{3} \cos (2 \theta-3 \varphi)-i \frac{1}{6} s^{3} r^{3} \sin (2 \theta-3 \varphi) & =\frac{s^{5} \cos 5 \varphi+i s^{5} \sin 5 \varphi}{r^{5}}
\end{aligned}
$$

By the equalities of real part and imaginary part of both sides of Eq. (13), the desire results hold. q.e.d.

Next, we obtain the solutions of limits (3) and (4).

Theorem 2 If the assumptions are the same as Theorem 1, then

$$
\begin{aligned}
& \cos 4 \theta \cdot \cos (s r \cos (\theta+\varphi)) \cdot \cosh (s r \sin (\theta+\varphi)) \\
&-\sin 4 \theta \cdot \sin (s r \cos (\theta+\varphi)) \cdot \sinh (s r \sin (\theta+\varphi)) \\
& \lim _{r \rightarrow 0} \frac{-\cos 4 \theta+\frac{1}{2} s^{2} r^{2} \cos (2 \theta-2 \varphi)}{r^{4}}=\frac{s^{4} \cos 4 \varphi}{24},
\end{aligned}
$$

and

$$
\begin{aligned}
&-\cos 4 \theta \cdot \sin (s r \cos (\theta+\varphi)) \cdot \sinh (s r \sin (\theta+\varphi)) \\
&-\sin 4 \theta \cdot \cos (s r \cos (\theta+\varphi)) \cdot \cosh (s r \sin (\theta+\varphi)) \\
& \lim _{r \rightarrow 0} \frac{+\sin 4 \theta-\frac{1}{2} s^{2} r^{2} \sin (2 \theta-2 \varphi)}{r^{4}}=\frac{s^{4} \sin 4 \varphi}{24} .
\end{aligned}
$$

Proof If $z=r e^{i \theta}, \lambda=s e^{i \varphi}$ in Eq. (10), then using Euler's formula and De Moivre's formula yields

$$
\lim _{r \rightarrow 0} \frac{e^{-i 4 \theta} \cos \left(s r e^{i(\theta+\varphi)}\right)-e^{-i 4 \theta}+\frac{1}{2} s^{2} r^{2} e^{i(-2 \theta+2 \varphi)}}{r^{4}}=\frac{s^{4} e^{i 4 \varphi}}{24} .
$$

Hence,

$$
\begin{aligned}
& (\cos 4 \theta-i \sin 4 \theta) \cdot \cos (s r \cos (\theta+\varphi)+i s r \sin (\theta+\varphi)) \\
& \lim _{r \rightarrow 0} \frac{-e^{-i 4 \theta}+\frac{1}{2} s^{2} r^{2} \cos (2 \theta-2 \varphi)-i \frac{1}{2} s^{2} r^{2} \sin (2 \theta-2 \varphi)}{r^{4}}=\frac{s^{4} \cos 4 \varphi+i s^{4} \sin 4 \varphi}{24} .
\end{aligned}
$$

By Eq. (6), we have

$$
\begin{aligned}
& (\cos 4 \theta-i \sin 4 \theta)[\cos (s r \cos (\theta+\varphi)) \cdot \cosh (s r \sin (\theta+\varphi)) \\
& -i \sin (s r \cos (\theta+\varphi)) \cdot \sinh (s r \sin (\theta+\varphi))]-\cos 4 \theta+i \sin 4 \theta \\
& \lim _{r \rightarrow 0} \frac{+\frac{1}{2} s^{2} r^{2} \cos (2 \theta-2 \varphi)-i \frac{1}{2} s^{2} r^{2} \sin (2 \theta-2 \varphi)}{r^{4}}
\end{aligned}=\frac{s^{4} \cos 4 \varphi+i s^{4} \sin 4 \varphi}{24} .
$$


Using the equalities of real part and imaginary part of both sides of Eq. (16) yields the desire results hold. q.e.d.

\section{Examples}

For the four types of limit problems discussed in this paper, we propose some examples and use Theorems 1 and 2 to obtain their solutions. In addition, Maple is used to calculate the approximations of these limits and their solutions for verifying our answers.

\subsection{Example}

In Theorem 1, let $s=3, \theta=4, \varphi=6$, then by Eq. (11), we have

$$
\begin{aligned}
& \cos 20 \cdot \sin (3 r \cos 10) \cdot \cosh (3 r \sin 10) \\
& \lim _{r \rightarrow 0} \frac{-3 r \cos 10+\frac{9}{2} r^{3} \cos 10}{r^{5}}=\frac{81 \cos 30}{40} .
\end{aligned}
$$

Using Maple to verify the correctness of Eq. (17) as follows:

$>$ evalf $(\operatorname{limit}((\cos (20) * \sin (3 * \mathrm{r} * \cos (10)) * \cosh (3 * \mathrm{r} * \sin (10)$ )$+\sin (20) * \cos (3 * r * \cos (10)) * \sinh (3 * r * \sin (10))-3 * r * \cos (10)$ $\left.\left.\left.+9 / 2 * \mathrm{r}^{\wedge} 3 * \cos (10)\right) / \mathrm{r}^{\wedge} 5, \mathrm{r}=0\right), 14\right)$;

$$
0.31235918602245
$$

$>\operatorname{evalf}(81 * \cos (30) / 40,14)$;

$$
0.31235918602235
$$

On the other hand, let $s=9, \theta=7, \varphi=3$ in Eq. (12), then

$$
\begin{aligned}
& \cos 35 \cdot \cos (9 r \cos 10) \cdot \sinh (9 r \sin 10) \\
& -\sin 35 \cdot \sin (9 r \cos 10) \cdot \cosh (9 r \sin 10) \\
& \lim _{r \rightarrow 0} \frac{+9 r \sin 25-\frac{243}{2} r^{3} \sin 5}{r^{5}}=\frac{19683 \cdot \sin 15}{40} .
\end{aligned}
$$
(18).

Also, we employ Maple to verify the correctness of Eq.

$>$ evalf $(\operatorname{limit}((\cos (35) * \cos (9 * \mathrm{r} * \cos (10)) * \sinh (9 * \mathrm{r} * \sin (10)$ )$-\sin (35) * \sin (9 * \mathrm{r} * \cos (10)) * \cosh (9 * \mathrm{r} * \sin (10))+9 * \mathrm{r} * \sin (25)-$ $\left.\left.\left.243 / 2 * \mathrm{r}^{\wedge} 3 * \sin (5)\right) / \mathrm{r}^{\wedge} 5, \mathrm{r}=0\right), 14\right)$;

\subsection{1}

>evalf(19683* $\sin (15) / 40,14)$;

$$
319.99038894531
$$

\subsection{Example}

In Theorem 2, if $s=2, \theta=4, \varphi=-3$, then using Eq. (14) yields

$$
\begin{aligned}
& \cos 16 \cdot \cos (2 r \cos 1) \cdot \cosh (2 r \sin 1) \\
& -\sin 16 \cdot \sin (2 r \cos 1) \cdot \sinh (2 r \sin 1) \\
& \lim _{r \rightarrow 0} \frac{-\cos 16+2 r^{2} \cos 14}{r^{4}}=\frac{2 \cos 12}{3} .
\end{aligned}
$$

Verify the correctness of Eq. (19) using Maple.

$>$ evalf $(\operatorname{limit}((\cos (16) * \cos (2 * \mathrm{r} * \cos (1)) * \cosh (2 * \mathrm{r} * \sin (1))-\mathrm{s}$ $\operatorname{in}(16) * \sin \left(2 * r^{*} \cos (1)\right) * \sinh \left(2 * r^{*} \sin (1)\right)-\cos (16)+2 * r^{\wedge} 2 * \cos$ $\left.\left.(14)) / \mathrm{r}^{\wedge} 4, \mathrm{r}=0\right), 14\right)$;

$$
0.56256930582166
$$

$>$ evalf $(2 * \cos (12) / 3,14)$;

$$
0.56256930582166
$$

Moreover, let $s=5, \theta=-3, \varphi=8$ in Eq. (15), we obtain

$$
\begin{aligned}
&-\cos 12 \cdot \sin (5 r \cos 5) \cdot \sinh (5 r \sin 5) \\
&+\sin 12 \cdot \cos (5 r \cos 5) \cdot \cosh (5 r \sin 5) \\
& \lim _{r \rightarrow 0} \frac{-\sin 12+\frac{25}{2} r^{2} \sin (22)}{r^{4}}=\frac{625 \sin 32}{24} .
\end{aligned}
$$

Also, we use Maple to verify the correctness of Eq. (20). $>$ evalf $(\operatorname{limit}((-\cos (12) * \sin (5 * \mathrm{r} * \cos (5)) * \sinh (5 * \mathrm{r} * \sin (5))+$ $\sin (12) * \cos (5 * \mathrm{r} * \cos (5)) * \cosh (5 * \mathrm{r} * \sin (5))-\sin (12)+25 / 2 * \mathrm{r}^{\wedge} 2$ $\left.\left.* \sin (22)) / \mathrm{r}^{\wedge} 4, \mathrm{r}=0\right), 14\right)$;

\subsection{3}

$>$ evalf( $625 * \sin (32) / 24,14)$;

$$
14.360069824003
$$

\section{Conclusions}

We know that theorems 1 and 2 is the main theoretical basis for solving the four types of limit problems discussed in this paper, and the complex function method in the theoretical derivation of this article occupies a pivotal position. Moreover, it can be seen that Maple plays an important role in assisting problem solving. We can even use Maple to design some limit problems and try to find the key to solving the problem. Future research will extend the tentacles to other areas of mathematics, and use Maple to solve these problems. These results can be used as teaching materials for Maple on education and research to enhance the connotations of calculus and engineering mathematics.

\section{REFERENCES}

[1] M. L. Abell, J. P. Braselton. Maple by Example (3rd ed.), Elsevier Academic Press, Massachusetts, 2005.

[2] N. Blachman, M. Mossinghoff. Maple V Quick Reference, Brooks/Cole, Boston, 1995

[3] R. M. Corless. Essential Maple, Springer-Verlag, New York, 1994 
[4] A. Heck. Introduction to Maple (3rd ed.), Springer-Verlag, New York, 2003

[5] R. Larson, B. H. Edwards. Calculus (10th edition), Brooks/Cole, Boston, 2014
[6] S. Y. Huang, C. -H. Yu. A study on some limit problems, (in Chinese), 2011 Nan Jeon General Education Symposium, Taiwan, pp.83-94, 2011

[7] C. -H. Yu. A study of limit problems, (in Chinese), Journal of Nan Jeon, Vol. 16, D2-1 14, 2013 